\title{
In-silico Drug Design, ADMET Screening, MM-GBSA Binding Free Energy of Some Chalcone Substituted 9-Anilinoacridines as HER2 Inhibitors for Breast Cancer
}

\author{
Kalirajan Rajagopal", Pandiselvi Arumugasamy, Gowramma Byran \\ Department of Pharmaceutical Chemistry, JSS College of Pharmacy [A Constituent College of JSS Academy of Higher Education \& \\ Research-(Deemed to Be University)], Udhagamandalam, Tamilnadu, India
}

Email address:

rkalirajan@ymail.com (K. Rajagopal), rkalirajan@jssuni.edu.in (K. Rajagopal)

${ }^{*}$ Corresponding author

\section{To cite this article:}

Kalirajan Rajagopal, Pandiselvi Arumugasamy, Gowramma Byran. In-silico Drug Design by Docking Studies, ADMET Screening, MMGBSA Binding Free Energy of Some Chalcone Substituted 9-Anilinoacridines as HER2 Inhibitors Targeting Breast Cancer. International Journal of Computational and Theoretical Chemistry. Vol. 7, No. 1, 2019, pp. 6-13. doi: 10.11648/j.ijctc.20190701.12

Received: January 23, 2019; Accepted: February 25, 2019; Published: March 18, 2019

\begin{abstract}
Due to their DNA-intercalating agents 9-aniliinoacridines play an important role as antitumor agents. A Series of some Chalcone substituted 9-aniliinoacridines 1a-x were designed for their anti-breast cancer activity. Molecular docking studies were performed by Glide module of Schrodinger suite-2016, targeted against Human epidermal growth factor receptor HER2 (PDB id-3PP0). In-silico ADMET screening by qikprop module and binding free energy by Prime-MMGBSA module also performed. Based on the binding affinity of the designed molecules with HER2 on the basis of GLIDE score and interaction patterns. Most of the compounds 1a-x have significant Glide scores when compared with standard anticancer drugs ledacrine and tamoxifen. Most of the Chalcone substituted 9-anilinoacridine derivatives 1a-x have good binding affinity with Glide score in the range of -5.32 to -9.37 compared with the standard ledacrine $(-5.23)$ and tamoxifen $(-3.78)$. The results reveals that, Chalcone substituted 9-amino acridines as HER2 inhibitor and the compounds, $1 \mathrm{~g}, \mathrm{f}, \mathrm{b}, \mathrm{h}, \mathrm{t}$, $\mathrm{u}$ with good Glide score may produce significant anti-breast cancer activity for further refinement.
\end{abstract}

Keywords: Docking Studies, Acridine, Chalcone, MM-GBSA, Antibreast Cancer, HER2

\section{Introduction}

Many chemotherapeutic agents still plays an important role in the fight against cancer. Especially, about 1 in 5 women affected by breast cancer. Human epidermal growth factor receptor HER 2 overexpression is present in $20-30 \%$ of the breast cancer. HER2 overexpression is associated with a more aggressive disease, higher recurrence rate, and shortened survival [1]. These type of breast cancers, grow and spread more aggressively. The benefit of anti-HER2 therapies are one of the most promising molecules for targeted therapy [2]. Human epidermal growth factor receptor-2 is membrane tyrosine kinase was over expressed and gene amplified in human breast cancers. So it is an important tumor cell proliferation and survival pathways [3]. Breast cancers have up to 25-50 copies of the HER2 gene, and up to $40-100$ fold increase in HER2 protein resulting in more than 2 million receptors expressed at the tumor cell surface (ERBB2 amplification in breast cancer analysed by fluorescence in situ hybridization [4].

In general, 9-aminoacridine derivatives are inhibiting DNA due to the ability of acridine nucleus to intercalate into DNA base pair. Presently available 9-aminoacridine derivatives like amsacrine and CI-921 a well-known anti-proliferative agent used in the treatment of acute leukaemia. They are biologically unstable because of the Amsacrine (m-AMSA) and CI-921 possess a methane sulfonyl and a methoxy function at $\mathrm{C}-1^{\prime}$ and $\mathrm{C}-3^{\prime}$ of the 9-anilino ring and readily undergo reversible oxidation either chemically or microsomally converted in to quinonediimine. More than $50 \%$ of the dose is excreted as the glutathione conjugate. To address these drawbacks of 9-aminoacridines, the effective strategy is to design some modified drugs to overcome these above problems. 
Acridines are more importance for their various pharmacological activities like antimicrobial [5], antimicrobial [6], anticancer [7-11], antimalarial [12, 13], anti-inflammatory [14], analgesic [15], antileishmanial [16], antinociceptive [17], acetyl cholinesterase inhibitors [17] and antiherpes [19] etc. The stuctural modification of acridines by the introduction of different substitutions were allowed expansion of research on the structure activity relationship to afford new insight into molecular interactions at the receptor level [20]. Similarly, chalcones are also be an important class of compounds with a wide range of biological activities [2123] like antimicrobial, anticancer etc.

The present research work by in-silico drug design gives knowledge about the new drug discovery by modifying the structure of the compounds for breast cancer activity and save time and money spending by wet lab. So after in-silico drug design, we will synthesize selected compounds with good docking score.

As part of our ongoing research on searching novel antitumor agents [24-27], we have designed some novel 9aminoacridine analogues bearing the chalcone residue on 9aminoacridine rings by molecular docking studies by using Schrodinger suit-2016. The results revealed that the newly designed chalcone substituted 9-aniliinoacridines exhibited significant inhibition with HER2 exhibit anti-breast cancer activity.

\section{Materials and Methods}

\subsection{Protein Preparation}

The Human epidermal growth factor receptor 2 (HER2) with co crystallized ligand (PDB ID: 3PP0, resolution 2.25 $\mathrm{A}^{\circ}$ ) was retrieved from protein data bank. protein preparation was performed by Protein preparation wizard module of Schrödinger suite 2016-2. Missing chain atoms are added by using prime module of Schrödinger suite 2016-2. Energy minimization of the protein structure was carried out using OPLS3 force field. A grid box was generated to defined the centroid of the active site for docking studies [28]

\subsection{Ligand Preparation}

The designed ligands (1a-x) were prepared by LigPrep module of Schrodinger suite 2016-2. 2D structures were converted to $3 \mathrm{D}$ structures, as well as energy minimization and optimized for their geometry, desalted and corrected for their chirality. The ionization and tautomeric states were generated between $\mathrm{pH}$ of 6.8 to 7.2 by using Epik module. The compounds 1a-x were minimized using Optimized Potentials for Liquid Simulations-3 (OPLS-3) force field in Schrodinger suit until a root mean square deviation of $2.0 \mathrm{~A}^{\circ}$ was achieved. A single low energy ring confirmation per ligand was generated and the optimized ligands were used for docking analysis.<smiles>[R]C=CC(=O)c1ccc(Nc2c3ccccc3nc3ccccc23)cc1</smiles>

\section{Chalcone substituted 9-anilinoacridine derivatives (1a-x)}

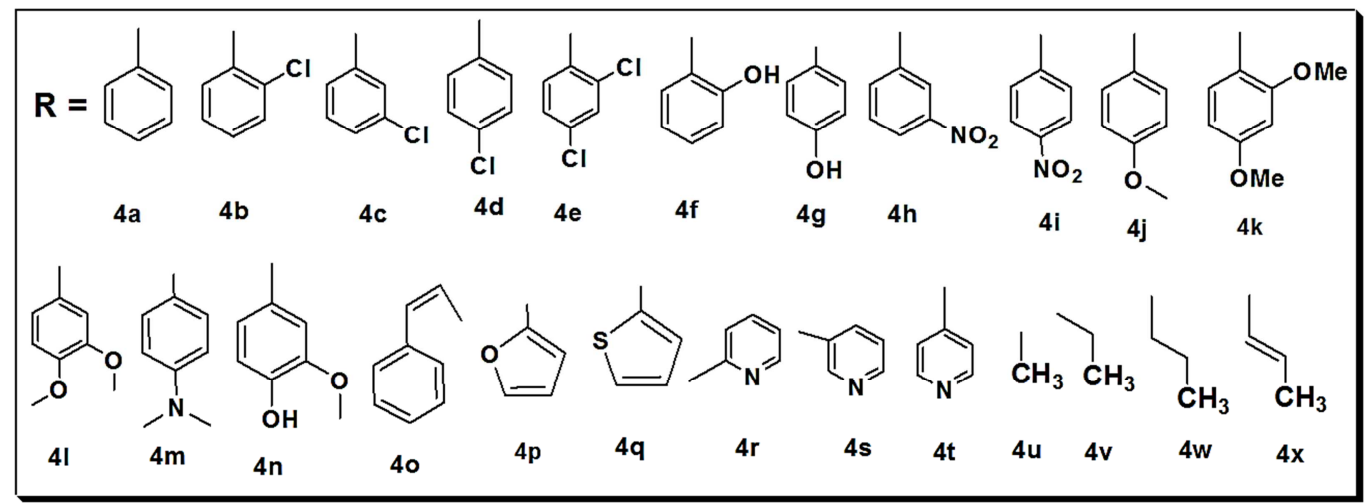

Figure 1. Structure of designed compounds (1a-x).

\subsection{Glide Ligand Docking}

The designed chalcone substituted 9-aminoacridines (1a-x) were docked in to catalytic pocket of HER2 protein (PDB ID:
3PP0) by Glide module of Schrödinger suite 2016-2. The favourable interactions between the receptor and ligands were scored by using Glide ligand docking module. Docking calculations were performed using extra precision (XP) mode 
and OPLS-3 force field. The flexible docking was performed for docking process in which automatically generates conformations for each ligand. This algorithm recognizes favourable hydrogen-bonding, hydrophobic, and electrostatic interactions, and penalizes steric clashes. Finally, the minimized poses were re-scored using Glide Score scoring function [29]. The XP-Glide score of the compounds were summarized and compared with the standard compound containing acridine ring ledacrine and the anti-breast cancer drug tamoxifen.

The in-silico ADMET properties of the proposed molecules were determined by qikprop module of Schrodinger suit-2016.

\subsection{Binding Free Energy Calculation by Using Prime/MM-GBSA Approach}

The binding free energies of ligand and receptor complex were computed by Molecular MechanicsGeneralized Born Surface Area (MM-GBSA) using the Prime module of Schrödinger suite 2016-2 which incorporates the OPLS3 force field and VSGB solvent model to search algorithms.

\section{Results and Discussion}

\subsection{Docking Studies}

The docking studies of the designed molecules (1a-x) to the protein active sites were performed by Glide module of Schrodinger suit-2016-2 for determining the binding affinities of the ligands. The designed compounds are docked towards the HER2 (3PP0) in order to ascertain their HER2 inhibition activity against breast cancer. The compounds $1 \mathrm{a}-\mathrm{x}$ were exhibited good affinity to the receptor when compared with standard acridine derivative with anticancer activity ledacrine and anti-breast cancer agent tamoxifen. The Glide scores of docking studies against HER2 inhibitor (PDB id 3PP0) are shown in the Table 1. From the docking results, the interactions are mainly lipophilic factors due to the presence of aromatic rings of acridine and heterocyclic rings. The ligand interactions of the compound $1 \mathrm{~g}$ with high Glide score $(-9.374)$ are mainly dominated in the region of LEU726 to PHE 864 residues which are the active site region (Figure 2). The compound $1 \mathrm{~g}$ is exhibited hydrogen bonding with LUU 807 (H-bond length 1.92) residues (Figure 3). The best docked poses of the compounds $1 \mathrm{~g}$ and $1 \mathrm{f}$ with high Glide score were shown in the Figure 4.

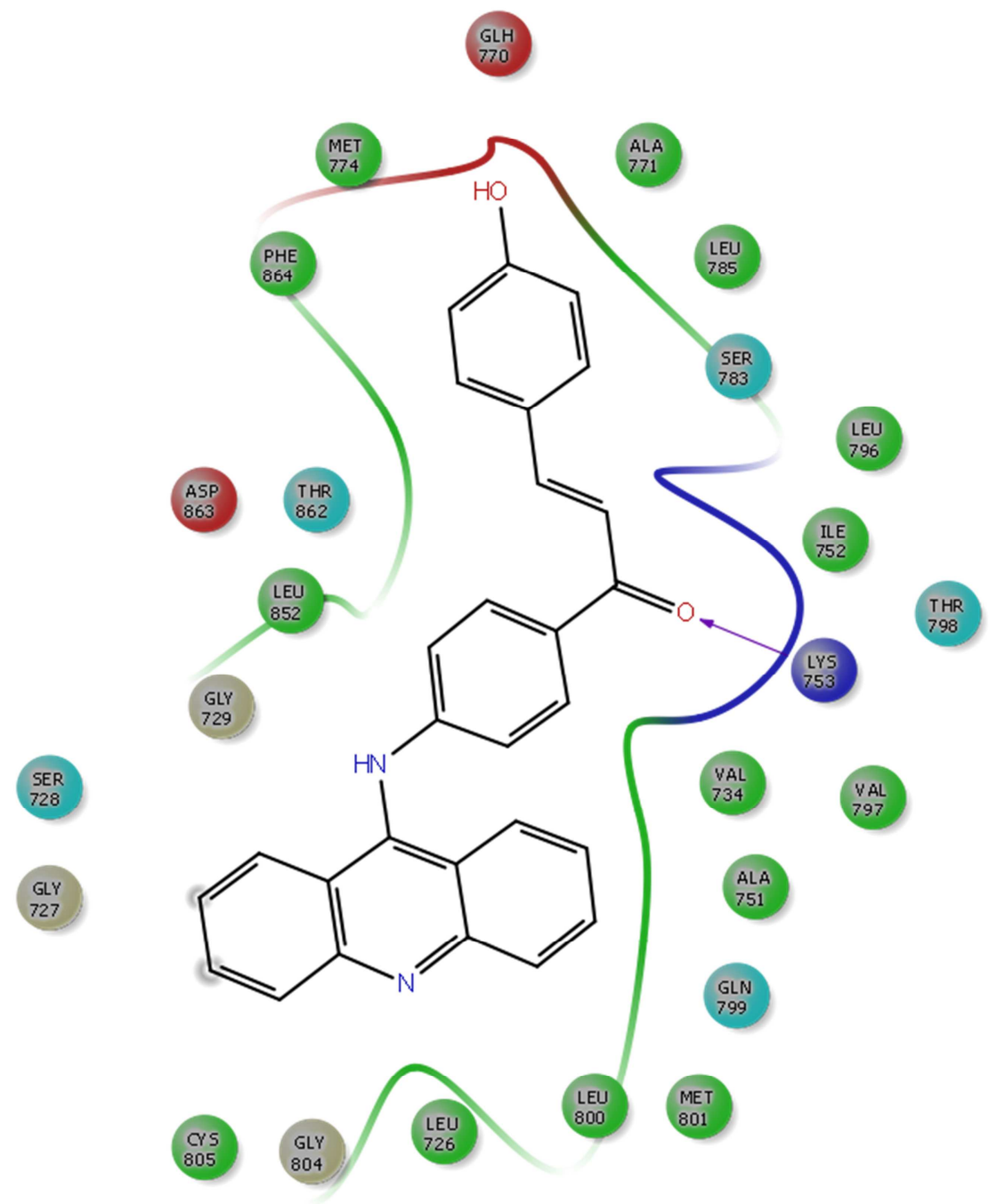

Figure 2. Ligand interaction of compound $1 g$ with HER2 (3PPO). 

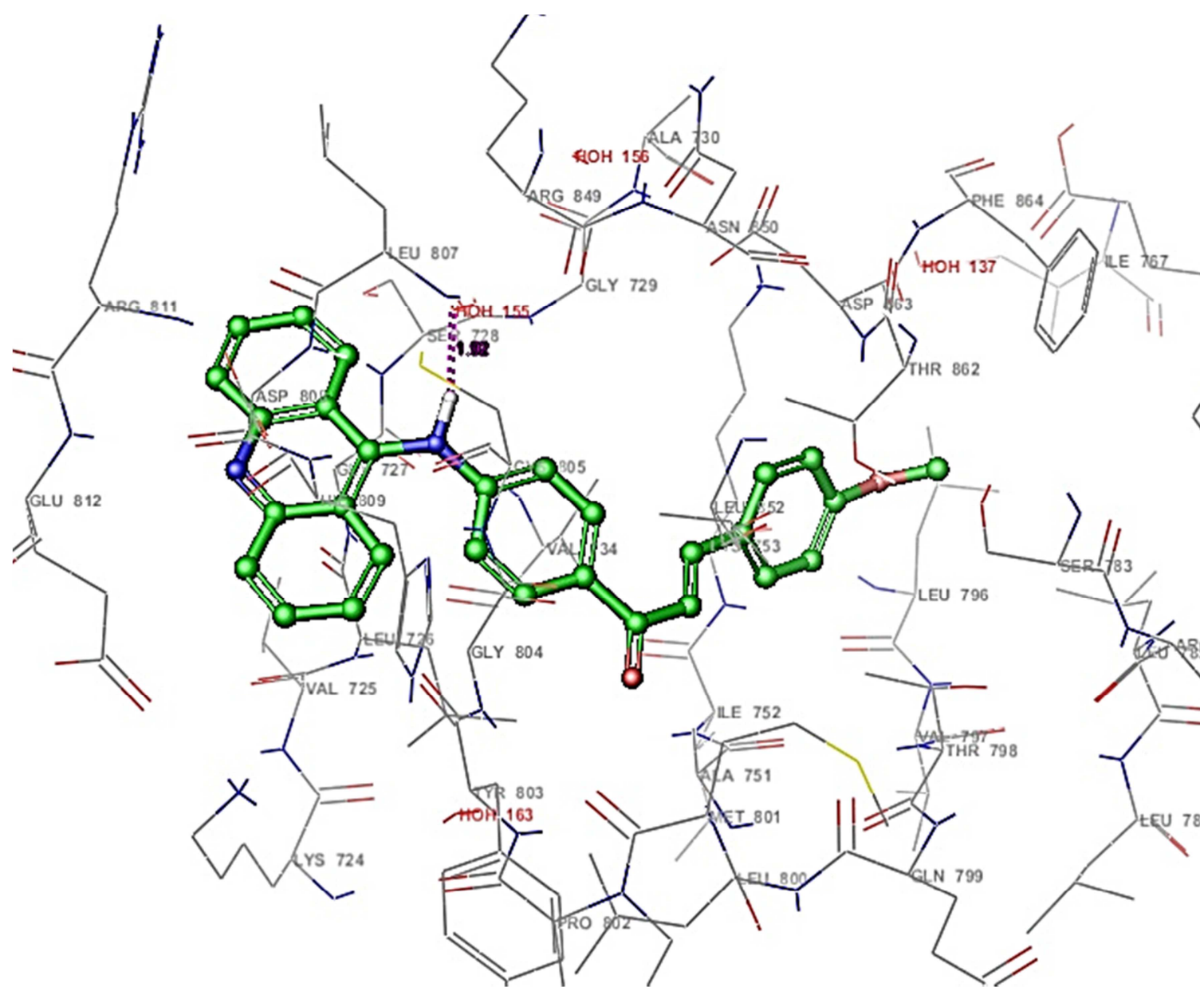

Figure 3. Hydrogen bonding affinity of docked compounds $1 g$ with HER2 (3PP0).

$1 \mathrm{~g}$

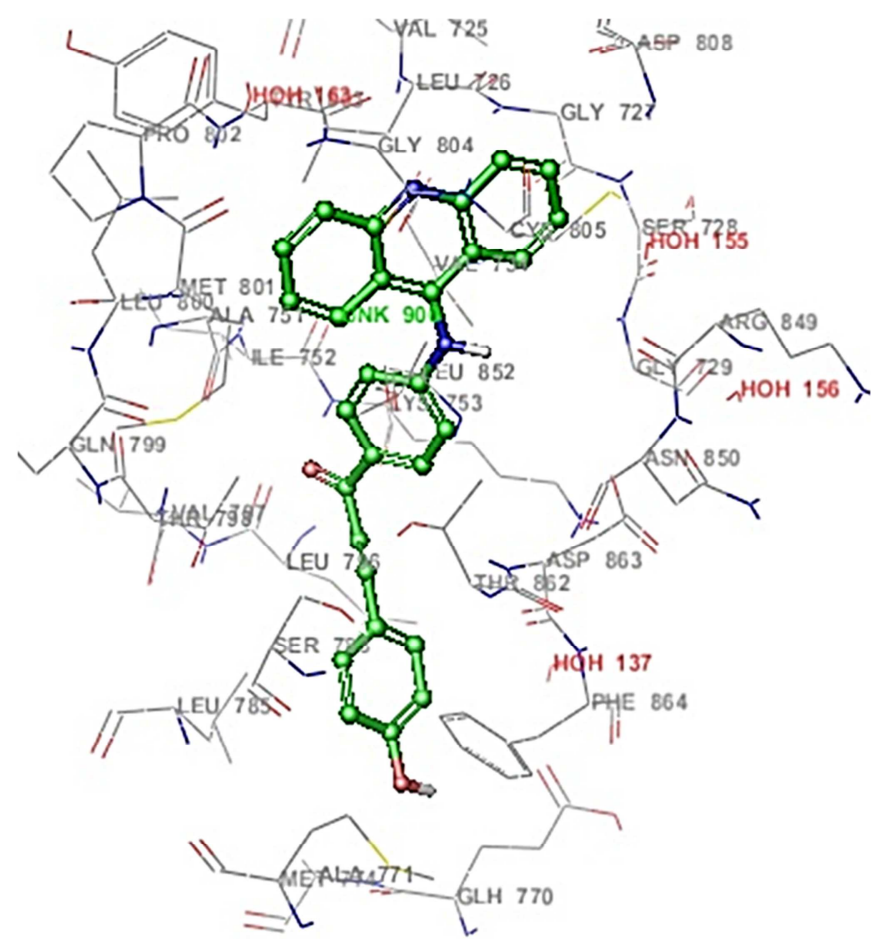

1f

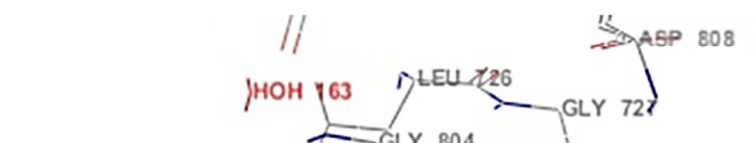

Figure 4. Best affinity mode of docked compounds $1 g$ and $1 f$ with HER2 (3PP0). 
Table 1. Docking results for Chalcone substituted 9-anilinoacridines (1a-x) with HER2 (3PP0).

\begin{tabular}{|c|c|c|c|c|c|c|c|}
\hline Compounds & GScore & Lipophilic EvdW & HBond & Electro & Low MW & Penalties & Rot Penal \\
\hline $1 \mathrm{~g}$ & -9.374 & -9.128 & -0.032 & 0.053 & -0.112 & 0 & 0.244 \\
\hline 1f & -9.358 & -9.127 & 0 & 0.037 & -0.112 & 0 & 0.244 \\
\hline $1 \mathrm{~b}$ & -8.586 & -9.277 & -0.112 & 0.072 & -0.05 & 1 & 0.181 \\
\hline $1 \mathrm{~h}$ & -8.558 & -9.159 & 0 & 0.05 & -0.015 & 1 & 0.216 \\
\hline $1 \mathrm{t}$ & -8.2 & -8.359 & 0 & -0.075 & -0.162 & 1 & 0.209 \\
\hline $1 \mathrm{u}$ & -8.108 & -7.656 & 0 & 0.037 & -0.372 & 0 & 0.283 \\
\hline $1 \mathrm{~s}$ & -7.977 & -8.676 & 0 & 0.052 & -0.162 & 1 & 0.209 \\
\hline $1 \mathrm{p}$ & -7.471 & -8.849 & 0 & -0.068 & -0.199 & 2.5 & 0.219 \\
\hline $1 \mathrm{v}$ & -7.459 & -8.097 & 0 & 0.033 & -0.325 & 1 & 0.329 \\
\hline $1 \mathrm{w}$ & -7.429 & -8.43 & 0 & 0.058 & -0.278 & 1.5 & 0.307 \\
\hline $1 \mathrm{c}$ & -7.005 & -9.743 & -0.32 & 0.003 & -0.05 & 4 & 0.181 \\
\hline 1a & -6.648 & -9.632 & 0 & 0.002 & -0.165 & 4 & 0.21 \\
\hline $1 \mathrm{x}$ & -6.5 & -8.474 & 0 & 0.074 & -0.285 & 2.5 & 0.31 \\
\hline 1n & -6.433 & -6.327 & -0.48 & 0.17 & -0.012 & 0 & 0.215 \\
\hline $1 \mathrm{e}$ & -6.357 & -9.77 & -0.157 & 0.031 & 0 & 4 & 0.157 \\
\hline $1 \mathrm{q}$ & -6.161 & -9.148 & 0 & -0.072 & -0.145 & 4 & 0.204 \\
\hline $1 \mathrm{j}$ & -6.13 & -6.103 & -0.7 & -0.101 & -0.065 & 0 & 0.23 \\
\hline $1 d$ & -6.044 & -6.687 & 0 & -0.03 & -0.05 & 0 & 0.181 \\
\hline $1 \mathrm{i}$ & -5.929 & -5.496 & -0.547 & 0.049 & -0.015 & 0 & 0.216 \\
\hline $1 \mathrm{~m}$ & -5.693 & -5.997 & -0.427 & -0.053 & -0.022 & 0 & 0.218 \\
\hline 10 & -5.515 & -5.562 & -0.404 & -0.067 & -0.078 & 0 & 0.234 \\
\hline $1 \mathrm{k}$ & -5.324 & -5.871 & 0 & -0.129 & 0 & 0 & 0.203 \\
\hline $1 \mathrm{r}$ & -3.797 & -9.151 & 0 & -0.043 & -0.162 & 6.4 & 0.209 \\
\hline 11 & -2.539 & -5.752 & -0.7 & -0.11 & 0 & 4 & 0.203 \\
\hline Lidacrine (std) & -5.23 & -3.95 & -0.83 & -0.12 & -0.15 & 0 & 0.1 \\
\hline Tamoxifen (std) & -3.787 & -4.179 & 0 & -0.262 & 0.42 & -0.023 & 0 \\
\hline
\end{tabular}

\subsection{Insilico ADMET Screening}

The ADMET properties of the designed ligands (1a-x) were determined in-silico by qikprop module of Schrödinger suite 2016-2. Molecular weight of the designed compounds between 352 1nd 460. Estimated no. of hydrogen bonds donors are in the range of 1-2. Estimated no. of hydrogen bonds acceptors are in the range of 3.5- 5. Predicted octanol/water partition coefficient are in the range of 4.9 to 7.2. Predicted brain/blood partition coefficient are in the range of -3.3 to -1.6 . Number of likely metabolic reactions of the compounds are in the range of 1-4. Prediction of binding to human serum albumin for the compounds are in the range of -0.8 to -1.4. Number of violations of Lipinski's rule of five is $0-1$. All the compounds have 90 to $100 \%$ Human Oral Absorption. So almost all the properties of the compounds are within the recommended values. The details of the ADMET properties for the compounds 1a-x are shown in the Table 2.

Binding free energy calculation using Prime/MM-GBSA

The stability of docking was also evaluated with MMGBSA free binding energy [30] which is related to post scoring approach for HER2 (PDB ID: 3PP0) target. The accuracy of docking is confirmed by the lowest energy poses predict by scoring function. The Glide scores are almost resembling to the experimental binding mode as determined by the X-ray crystallography. The Glide score and MMGBSA free energy values are obtained by the docking of ligands in to the binding pocket. The details of the MMGBSA free binding energy for the ligands 1a-x are shown in the Table 3.

Table 2. Insilico ADMET screening for compounds 1a-x.

\begin{tabular}{llllllllll}
\hline Compounds & mol MW & Donor HB & Accpt HB & QPlogP o/w & QP log BB & \# metab & $\begin{array}{l}\text { QPlog } \\
\text { Khsa }\end{array}$ & $\begin{array}{l}\text { \%Human Oral } \\
\text { Absorption }\end{array}$ & $\begin{array}{l}\text { Rule of } \\
\text { Five }\end{array}$ \\
\hline 1a & 400.479 & 1 & 3.5 & 6.302 & -0.498 & 2 & 1.224 & 100 & 1 \\
1b & 434.924 & 1 & 3.5 & 6.751 & -0.357 & 2 & 1.333 & 100 & 1 \\
1c & 434.924 & 1 & 3.5 & 6.8 & -0.336 & 1 & 1.345 & 100 & 1 \\
1d & 434.924 & 1 & 3.5 & 6.801 & -0.337 & 2 & 1.346 & 100 & 1 \\
1e & 469.369 & 1 & 3.5 & 7.238 & -0.192 & 1 & 1.452 & 100 & 1 \\
1f & 416.478 & 2 & 4.25 & 5.552 & -1.06 & 3 & 0.996 & 100 & 1 \\
1g & 416.478 & 2 & 4.25 & 5.505 & -1.164 & 3 & 1.005 & 100 & 1 \\
1h & 445.476 & 1 & 4.5 & 5.595 & -1.667 & 2 & 1.172 & 90.76 & 1 \\
1i & 445.476 & 1 & 4.5 & 5.607 & -1.676 & 2 & 1.175 & 90.831 & 1 \\
$1 \mathrm{j}$ & 430.505 & 1 & 4.25 & 6.404 & -0.576 & 3 & 1.235 & 100 & 1 \\
\hline
\end{tabular}




\begin{tabular}{|c|c|c|c|c|c|c|c|c|c|}
\hline Compounds & mol MW & Donor HB & Accpt HB & $\mathrm{QP} \log P \mathrm{o} / \mathrm{w}$ & QP $\log B B$ & \# metab & $\begin{array}{l}\text { QPlog } \\
\text { Khsa } \\
\end{array}$ & $\begin{array}{l}\text { \%Human Oral } \\
\text { Absorption }\end{array}$ & $\begin{array}{l}\text { Rule of } \\
\text { Five }\end{array}$ \\
\hline $1 \mathrm{k}$ & 460.531 & 1 & 5 & 6.532 & -0.659 & 4 & 1.252 & 100 & 1 \\
\hline 11 & 460.531 & 1 & 5 & 6.582 & -0.661 & 4 & 1.268 & 100 & 1 \\
\hline $1 \mathrm{~m}$ & 443.547 & 1 & 4.5 & 6.722 & -0.635 & 3 & 1.387 & 100 & 1 \\
\hline 1n & 446.504 & 2 & 5 & 5.742 & -1.044 & 4 & 1.015 & 100 & 1 \\
\hline 10 & 426.517 & 1 & 3.5 & 7.011 & -0.659 & 2 & 1.429 & 100 & 1 \\
\hline $1 \mathrm{p}$ & 390.44 & 1 & 4 & 5.673 & -0.49 & 3 & 0.959 & 100 & 1 \\
\hline $1 \mathrm{q}$ & 406.501 & 1 & 3.5 & 6.213 & -0.391 & 3 & 1.145 & 100 & 1 \\
\hline $1 \mathrm{r}$ & 401.467 & 1 & 4.5 & 5.633 & -0.718 & 3 & 0.985 & 100 & 1 \\
\hline $1 \mathrm{~s}$ & 401.467 & 1 & 5 & 5.354 & -0.802 & 3 & 0.878 & 100 & 1 \\
\hline $1 \mathrm{t}$ & 401.467 & 1 & 5 & 5.35 & -0.802 & 3 & 0.877 & 100 & 1 \\
\hline $1 \mathrm{u}$ & 338.408 & 1 & 3.5 & 4.956 & -0.335 & 3 & 0.827 & 100 & 0 \\
\hline $1 \mathrm{v}$ & 352.435 & 1 & 3.5 & 5.342 & -0.416 & 3 & 0.954 & 100 & 1 \\
\hline $1 \mathrm{w}$ & 366.462 & 1 & 3.5 & 5.729 & -0.496 & 3 & 1.078 & 100 & 1 \\
\hline $1 \mathrm{x}$ & 364.446 & 1 & 3.5 & 5.651 & -0.492 & 3 & 1.027 & 100 & 1 \\
\hline $\begin{array}{l}\text { Recommended } \\
\text { values }\end{array}$ & $130-725$ & $0-6$ & $2-20$ & $2-6.5$ & $-3-1.2$ & $1-8$ & $-1.5-1.5$ & $7-200$ & $0-4$ \\
\hline
\end{tabular}

MW- Molecular weight of the molecule,

donorHB - Estimated number of hydrogen bonds that would be donated by the solute to water molecules in an aqueous solution. accptHB- Estimated number of hydrogen bonds that would be accepted by the solute from water molecules in an aqueous solution QPlogPo/w - Predicted octanol/water partition coefficient.

QP $\log$ BB Predicted brain/blood partition coefficient

\#metab- Number of likely metabolic reactions.

QPlogKhsa- Prediction of binding to human serum albumin.

RuleOfFive Number of violations of Lipinski's rule of five.

\%Human- Oral absorption- Predicted human oral absorption on 0 to $100 \%$ scale.

Table 3. Binding free energy calculation for compounds la-x using Prime/MM-GBSA approach.

\begin{tabular}{lllllll}
\hline Compounds & $\Delta$ Gbind (Kcal/mol) & $\Delta$ Gbind Coulomb & $\Delta$ Gbind Vander & $\Delta$ Gbind HBond & $\Delta$ Gbind covalent & $\Delta$ Gbind Lipophilic \\
\hline 1a & -63.2916 & -35.6655 & -37.2151 & -2.94571 & -5.15878 & -26.9411 \\
1b & -64.4452 & -26.1298 & -39.7237 & -0.43671 & -8.03151 & -28.5308 \\
1c & -40.3856 & 20.71722 & -35.1217 & 5.732459 & -17.1177 & -26.4266 \\
1d & -64.1664 & -3.75509 & -42.1457 & -0.53399 & -5.19287 & -25.9174 \\
1e & -68.0454 & 9.404431 & -53.6695 & 1.296063 & -12.0695 & -26.2351 \\
1f & -60.9029 & -20.1311 & -30.3334 & -0.66922 & -2.12714 & -20.1654 \\
1g & -64.5958 & -10.3487 & -39.6925 & 3.270054 & -17.5304 & -26.6284 \\
1h & -67.3007 & -26.1181 & -39.2795 & -1.0628 & -9.15781 & -25.5037 \\
1i & -72.0653 & -10.1229 & -46.8267 & -0.95492 & -6.14237 & -22.722 \\
1j & -65.5068 & -11.8431 & -46.2886 & -1.38587 & -7.0343 & -25.0214 \\
1k & -73.6077 & -13.0051 & -39.0165 & 1.70326 & -17.0842 & -29.4083 \\
11 & -79.4697 & -27.2758 & -50.7698 & -0.4232 & -4.23311 & -31.2384 \\
1m & -68.871 & -10.5547 & -38.6772 & 1.026628 & -16.6679 & -24.607 \\
1n & -43.6982 & -6.83597 & -43.4849 & 2.434836 & 13.21079 & -26.1138 \\
10 & -73.6327 & -18.4178 & -45.0856 & 1.502527 & -11.9557 & -33.0445 \\
1p & -74.1282 & -18.4891 & -51.5734 & -1.35663 & -7.11058 & -28.6394 \\
1q & -37.5453 & 1.922893 & -36.9764 & 0.10371 & -13.3517 & -17.0213 \\
1r & -48.314 & -34.3285 & -35.7612 & -0.3345 & -4.86448 & -19.2435 \\
1s & -61.6737 & -39.0154 & -56.924 & -5.6718 & 16.7104 & -21.567 \\
1t & -55.7057 & -39.2176 & -41.3566 & -1.44176 & -6.81391 & -21.022 \\
1u & -72.5252 & -19.4196 & -53.6654 & 0.046481 & 3.02907 & -31.5128 \\
1v & -46.7637 & -22.3516 & -35.7654 & 1.073849 & -3.88373 & -17.5289 \\
1w & -70.0558 & -4.41536 & -50.4707 & 0.266061 & -8.52899 & -29.1382 \\
1x & -63.193 & -8.45533 & -47.8544 & 0.845721 & 3.264462 & -26.7074 \\
\hline & & & & &
\end{tabular}

\section{Conclusion}

9-Aminoacridines are exhibited various biological activities. The molecular docking study revealed that chalcone substituted 9-aminoacridines were showed better alignment at active site by interacting with many amino acid residues. The in-silico method adopted in the present study helped to identify the lead molecules. On this basis, we are recently demonstrated that diverse compounds of the chalcone substituted 9- 
anilinoacridine series exerted HER2 inhibition as anti-breast cancer activity. The present study clearly demonstrated that many derivatives of the chalcone substituted 9-anilinoacridine family may exert interesting antitumour activity. The compounds $1 \mathrm{~g}, \mathrm{f}, \mathrm{b}, \mathrm{h}, \mathrm{t}$, u may have significant anti-breast cancer activity with therapeutic potentials and are likely to be useful as drugs after further refinement.

\section{Acknowledgements}

We thank our Vice Chancellor Dr. B. Suresh, JSS Adademy of Higher education \& Research, Mysuru, our principal Dr. S. P. Dhanabal, JSS College of pharmacy, Ooty for the technical support.

\section{References}

[1] Zahi Mitri; Tina Constantine; and Ruth O'Regan., The HER2 Receptor in Breast Cancer: Pathophysiology, Clinical Use, and New Advances in Therapy. Chemotherapy Research and Practice. 2012, doi: 10. 1155/2012/743193.

[2] Elda Tagliabue; Andrea Balsari; Manuela Campiglio; Serenella M. Pupa. HER2 as a target for breast cancer therapy, Expert Opin. Biol. Ther. 2010, 10 (5), 711-724.

[3] Kallioniemi, O. P., Kallioniemi, A., Kurisu, W., Thor, A., Chen, L. C., Smith, H. S., Waldman, F. M., Pinkel, D., Gray, J. W. ERBB2 amplification in breast cancer analysed by fluorescence in situ hybridization Proc. Natl. Acad. Sci. USA. 1992, 89 (12), 5321-535.

[4] Carolina Gutierrez, M. D., Rachel Schiff, Ph. D. HER 2: Biology, Detection, and Clinical Implications. Arch Pathol Lab Med. 2011, 135 (1), 55-62.

[5] Nadaraj, V.; Selvi, S. T.; Mohan, S. Microwave-induced synthesis and anti-microbial activities of 7, 10, 11, 12tetrahydrobenzo [c]acridin-8 (9H)-one derivatives. Eur. J. Med. Chem. 2009, 44, 976-982.

[6] Dickens, B. F.; Weglicki, W. B.; Boehme, P. A.; Mak, I. T. Antioxidant and lysosomotropic properties of acridinepropranolol: protection against oxidative endothelial cell injury. J. Mol. Cell Cardiol. 2002, 34, 129-137.

[7] Rouvier, C. S.; Barret, J. M.; Farrell, C. M.; Sharples, D.; Hill, B. T.; Barbe, J. Synthesis of 9-acridinyl sulfur derivatives: sulfides, sulfoxides and sulfones. Comparison of their activity on tumour cells. Eur. J. Med. Chem. 2004, 39, 1029-1038.

[8] Rastogi, K.; Chang, J. Y.; Pan, W. Y.; Chen, C. H.; Chou, T. C.; Chen L. T.; et al., Antitumor AHMA linked to DNA minor groove binding agents: synthesis and biological evaluation. $J$. Med. Chem. 2002, 45, 4485-4493.

[9] Kalirajan, R.; Vivek kulshrestha; Sankar, S.; Jubie, S. Docking studies, synthesis, characterization of some novel oxazine substituted 9-anilinoacridine derivatives and evaluation for their anti oxidant and anticancer activities as topo isomerase II inhibitors. Eur. J. Med. Chem. 2012, 56, 217-224.

[10] Bacherikov, V. A.; Chou, T. C.; Dong, H. J.; Chen, C. H.; Lin, Y. W.; Tsai, T. J. Potent antitumor N-mustard derivatives of 9anilinoacridine, synthesis and antitumor evaluation. Bioorg. Med. Chem. Lett. 2004, 14, 4719-4722.
[11] Kalirajan, R.; Muralidharan, V.; Jubie, S.; Gowramma, B.; Gomathy, S.; Sankar, S.; Elango, K. Synthesis of some novel pyrazole substituted 9-anilino acridine derivatives and evaluation for their antioxidant and cytotoxic activities. $J$. Heterocycl. chem. 2012, 49, 748-754.

[12] Gamage, S. A.; Tepsiri, N.; Wilairat, P.; Wojcik, S. J.; Figgitt, D. P.; Ralph, R. K. et al. Synthesis and invitro evaluation of 9anilino-3, 6-diaminoacridines active against a multidrugresistant strain of the malaria parasite plasmodium falciparum. J. Med. Chem. 1994, 37, 1486-1494.

[13] Anderson, M. O.; Sherrill. J.; Madrid, P. B.; Liou, A. P.; Weisman, J. L.; De Risib, J. L.; et al. Parallel synthesis of 9aminoacridines and their evaluation against chloroquineresistant Plasmodium falciparum. Bioorg. Med. Chem. 2006, $14,334-43$.

[14] Chen, Y. L.; Lu, C. M.; Chen, I. L.; Tsao, L. T.; Wang, J. P. Synthesis and antiinflammatory evaluation of 9anilinoacridine and 9-phenoxyacridine derivatives. J. Med. Chem. 2002, 45 (21), 4689-4694.

[15] Sondhi, S. M.; Johar, M.; Nirupama, S.; Sukla, R.; Raghubir, R. S. G. Synthesis of sulpha drug acridine derivatives and their evaluation for anti-anflammatory, analgesic and anticancer acvity. Indian J. Chem. 2002, 41B, 2659-2666.

[16] Gamage, S. A.; Figgitt, D. P.; Wojcik, S. J.; Ralph, R. K.; Ransijn, A.; Mauel, J.; et al. Structure-activity relationships for the antileishmanial and antitrypanosomal activities of 1'substituted 9-anilinoacridines. J. Med. Chem., 1997, 40, 26342642.

[17] Llama, E. F.; Campo, C. D.; Capo, M.; Anadon, M. Synthesis and antinociceptive activity of 9-phenyl-oxy or 9-acyl-oxy derivatives of xanthene, thioxanthene and acridine. Eur. $J$. Med. Chem. 1989, 24, 391-396.

[18] Recanatini, M.; Cavalli, A.; Belluti, F.; Piazzi, L.; Rampa, A.; Bisi, A.; et al. SAR of 9-amino-1, 2, 3, 4-tetrahydroacridinebased acetyl cholinesterase inhibitors: synthesis, enzyme inhibitory activity, QSAR, and structure-based CoMFA of tacrine analogues. J. Med. Chem. 2000, 43 (10), 2007-2018.

[19] Goodell, J. R.; Madhok, A. A.; Hiasa, H.; Ferguson, D. M. Synthesis and evaluation of acridine- and acridone-based antiherpes agents with topoisomerase activity. Bioorg. Med. Chem. 2006, 14, 5467-5480.

[20] Harrison, R. J., Cuesta, J., Chessari, G., Read, M. A., Basra, S. K., Reszka, A. P. et al. Trisubstituted acridine derivatives as potent and selective telomerase inhibitors. J. Med. Chem. 2003, 46, 4463-4476.

[21] Guodong Shen, Dingben Chen, Yiliang Zhang, Manman Sun, Kai Chen, Cong Jin, Kaining Li, Weiliang Bao. Synthesis of benzoxazine and 1,3-oxazine derivatives via ligand-free copper (I)-catalyzed one-pot cascade addition/cyclization reaction. Tetrahedron. 2012; 68, 166-72.

[22] Kalirajan, R.; Sivakumar, S. U.; Jubie, S.; Gowramma, B.; Suresh, B. Synthesis and biological evaluation of some heterocyclic derivatives of chalcones. Int. J. Chemtech. Res. 2009, 1, 27-34.

[23] Kalirajan, R.; Pandiselvi, M.; Sankar, S.; Gowramma, B. Molecular Docking Studies and Insilico ADMET Screening of Some Novel Chalcone Substituted 9-Anilinoacridines as Topoisomerase II Inhibitors, SF J. Pharm. Anal. Chem., 2018; 1 (1): 1004. 
[24] Kalirajan, R., Mohammed rafick, M. H., Jubie, S., Sankar, S. Docking studies, synthesis, characterization and evaluation of their antioxidant and cytotoxic activities of some novel isoxazole substituted 9-anilinoacridine derivatives. The Sci. World J. 2012, doi: 10. 1100/2012/165258.

[25] Rajagopal Kalirajan; Muralidharan, V.; Selvaraj Jubie and Sankar. S. Microwave assisted Synthesis, Characterization and Evaluation for their Antimicrobial Activities of Some Novel pyrazole substituted 9-Anilino Acridine Derivatives. Int. J. Health and Allied Sci. 2013, 2 (2), 81-87.

[26] Kalirajan, R; Gowramma, B; Jubie, S and Sankar S. Molecular Docking Studies and In-silico ADMET Screening of Some Novel Heterocyclic Substituted 9-Anilinoacridines as Topoisomerase II Inhibitors. JSM Chem, 2017, 5 (1), 10391044.

[27] Kalirajan, R; Vivek kulshrestha; Jubie, S and Sankar S. Synthesis, Characterization and Evaluation for Antitumour Activity of Some Novel Oxazine Substituted 9-
Anilinoacridines and their 3D-QSAR Studies. Indian $J$. Pharm. Sci. 2018, 80 (5), 921-929.

[28] Kalirajan, R; Sankar, S; Jubie, S and Gowramma, B. Molecular Docking studies and in-silico ADMET Screening of Some novel Oxazine substituted 9-Anilinoacridines as Topoisomerase II Inhibitors. Indian J. Pharm. Educ. Res., 2017, 51 (1), 110-115.

[29] Halperin, I.; Ma, B.; Wolfson, H.; Nussinov, R. Principles of Docking: An Overview of Search Algorithms and a Guide to Scoring Functions. PROTEINS: St Fun Gen. 2002, 47, 40943.

[30] Naga Srinivas Tripuraneni, Mohammed. Afzal Azam, Pharmacophore modelling, 3D-QSAR and docking study of 2phenylpyrimidine analogues as selective PDE4B inhibitors, $J$ Theoretical Biology, 2016, 394, 117-126. 